\title{
Application of OSPF Routing Protocol in Network Security
}

\author{
Yuntao Duan ${ }^{1}$, Siping Hü, ${ }^{*}$, Shejie Lu², Sheng Yang ${ }^{2}$ \\ ${ }^{1}$ Nanyang Institute of Technology, Nanyang, 473000, China \\ ${ }^{2}$ Hubei University of Science and Technology, Xianning, 430070, China \\ *E-mail: zjlg0001@126.com
}

Keywords: OSPF; Internet; Network Planning; Network Security

\begin{abstract}
This paper combines the specific needs of the domestic hotel industry, puts forward the requirements and feasibility of network planning and design, designs a specific network implementation plan, and uses technologies such as VLAN, OSPF, redundant gateways, and ACL access control lists to implement the network in the network solution. Normal communication and network security are tested and adjusted after the completion of the construction of the network. This study has a certain reference value for the development direction of the future hotel network.
\end{abstract}

\section{Introduction}

The era is developing, the society is advancing, and the Internet industry is developing especially fast. The Chinese hotel industry is also facing reforms along with the development of the Internet. The informationization of the hotel industry has been inevitable. The network has penetrated into all aspects of people's lives. Modern hotels should comply with customer needs and development of the times and integrate the hotel's original services such as accommodation, entertainment, and catering services into a network system to provide customers with more intelligent services. The modernization and informationization of the hotel requires its construction of a fully functional, easy-to-use and secure network system.

After such a network, on the one hand to provide customers with the need for business meetings, voice calls, video conferencing and other Internet, to provide customers with reliable, secure, intimate communication services; on the other hand, the hotel management system into the hotel In the network system, thus effectively improving the work efficiency and service quality of the hotel staff. Through the establishment of intelligent office, e-commerce and other network functions, to improve the hotel's service quality, to provide customers with a comfortable experience.

In Europe and the United States, due to the social system, European and American

China is still a developing country, and the pressure on the people is greater. More hotels are providing places for people to travel, so China has a lot of business hotels. With the development of the country, the quality of life of the people is getting better and better, and more and more people are willing to go out and relax and relax themselves. This places higher demands on the services of the Chinese hotel industry.

To build a brand new hotel network in response to the times requires the hotel to design the network construction as the infrastructure during the construction of the building, so that the hotel's multiple systems, such as the access control system, reservation system, etc. are all integrated on the same network, so that such a security A smooth, flexible network replaces multiple networks in the past, which can improve hotel service quality and reduce construction costs.

\section{Related technologies}

\subsection{Virtual Local Area Network (VLAN)}

Virtual Local Area Network (VLAN) is a technology that logically divides the physical devices in a LAN into new LANs, thereby cutting the broadcast domain and allowing the data in the virtual switching group to be exchanged [4]. 
The VLAN is used in Layer 2 and Layer 3 of the OSI Layer 7 model. A VLAN is a broadcast domain. Data exchange between different VLANs is accomplished through the router's routing function. VLAN technology is very convenient. The use of virtual LAN technology facilitates management of network devices. It isolates broadcast domains and improves network security.

\subsection{Spanning Tree Protocol (STP)}

The STP spanning tree protocol is mainly used to prevent loops in the network. By establishing a tree topology, the loop is reduced and the communication line can be redundant. The basic principle of STP is to determine the network topology by passing protocol packets such as BRIDGE PROTOCOL DATA UNIT (BPDU for short) between switches.

Spanning Tree Protocol (STP) can also be said to be a network security technology. It can eliminate network loopback caused by certain events and solve the broadcast storm problem formed in the network. STP also provides the possibility of providing a backup connection for the network, which can be combined with SDH protection to form dual protection of the Ethernet ring.

The HSRP router uses the HELLO packet to monitor the status of the router in the same group. When the router does not receive the HELLO packet within a certain period of time, it considers the sensation of the router failure. Then the backup router becomes the active router. The other routers in the backup group select one router based on the priority. Backup routers, so there is only one active router and backup router in a group.

\subsection{Open Shortest Path First (OSPF)}

OSPF opens the shortest path first and is a link state routing protocol. Unlike distance vector routing protocols that work according to the path that other routers tell, link-state routers obtain first-hand information from other routers. Each router generates some information about itself, the local direct link status, and all Even neighbor information (LSA). This information is forwarded from one router to another. Each router copies a piece of information but never changes the information. The ultimate goal is that each router has the same related network information, and each router independently calculates its own optimal path.

OSPF was only developed in 1989, and OSPF was developed to overcome the shortcomings of the RIP protocol. The main feature of OSPF is that it uses the link state protocol instead of the distance vector protocol. The characteristics of OSPF are:

1) Use the concept of regionalization, which can effectively reduce the CPU and memory usage of the algorithm and build a hierarchical network topology.

2) Supports classless routing, VLSM, CIDR.

3) Supports no size restrictions, arbitrary metrics.

4) Partially updated.

5) Equivalent load balancing for multiple paths is supported.

6) Use multicast address updates (224.0.0.5 and 224.0.0.6).

7) Support certification.

\section{Hotel Network Construction Implementation Plan}

\subsection{Divide VLAN}

Different departments of the hotel have different natures of work. Data traffic needs to be separated. Each department is divided into different LVANs using VLAN technology. Rooms are divided into VLANs by floors. The use of VLAN technology has the following advantages:

Can prevent broadcast storms. Dividing VLANs can reduce broadcast traffic on the hotel network, ensuring the smooth flow of customers' Internet access and giving customers a high-speed Internet experience.

Ensure the security of the hotel network. In a switched network, different VLANs cannot communicate directly. They need to go through the access layer, convergence layer, and core layer. The three-layer network can be verified only after communication. This ensures the security of the 
hotel network and gives customers Brought a safe and secure Internet environment.

VLAN technology is used to logically divide a planar network into multiple subnets, reducing traffic on the network, improving performance, and improving the efficiency of network management.

The VLAN division information is as follows:

Different VLANs are assigned on the switch according to different departments and ports are allocated for each VLAN. Each floor of a hotel room network belongs to a VLAN.

Take the Administration Department as an example, the VLAN configuration is as follows:

Switch>en

Switch\#conf $\mathrm{t}$

Switch(config)\#vlan 100

Switch(config-vlan)\#exit

Switch(config)\#int f0/1

Switch(config-if)\#sw mode access

Switch(config-if)\#sw access vlan 100

Figure 1 Divide VLAN

\subsection{Setting up a DHCP service}

The hotel network belongs to the campus network. However, the network is large and the network is complicated. Therefore, manually assigning addresses is not recommended. This requires a lot of manpower and the cost is too high. Therefore, we set up a DHCP server to dynamically allocate addresses for hosts.

Set up a DHCP server for each VLAN of the work network and room network.

Take VLAN100 as an example, define the address pool VLAN100 related commands as follows:

Switch>en

Switch\#

Switch\#conf $\mathrm{t}$

Enter configuration commands, one per line. End with CNTL/Z.

Switch(config)\#ip dhcp pool xz

Switch(dhcp-config)\#network 10.1.3.0 255.255.255.0

Switch(dhcp-config)\#default-router 10.1.3.254

Switch(dhcp-config)\#dns-server 8.8.8.8

Switch(dhcp-config)\#exit

Figure 2 Configure DHCP service

\section{Network Connectivity Commissioning}

\subsection{Inter-VLAN Communication Test}

Select one PC0 (10.1.3.1) of the work subnet administration department to PING another host PC1 (10.1.4.1). The test result is shown in Figure 4: 


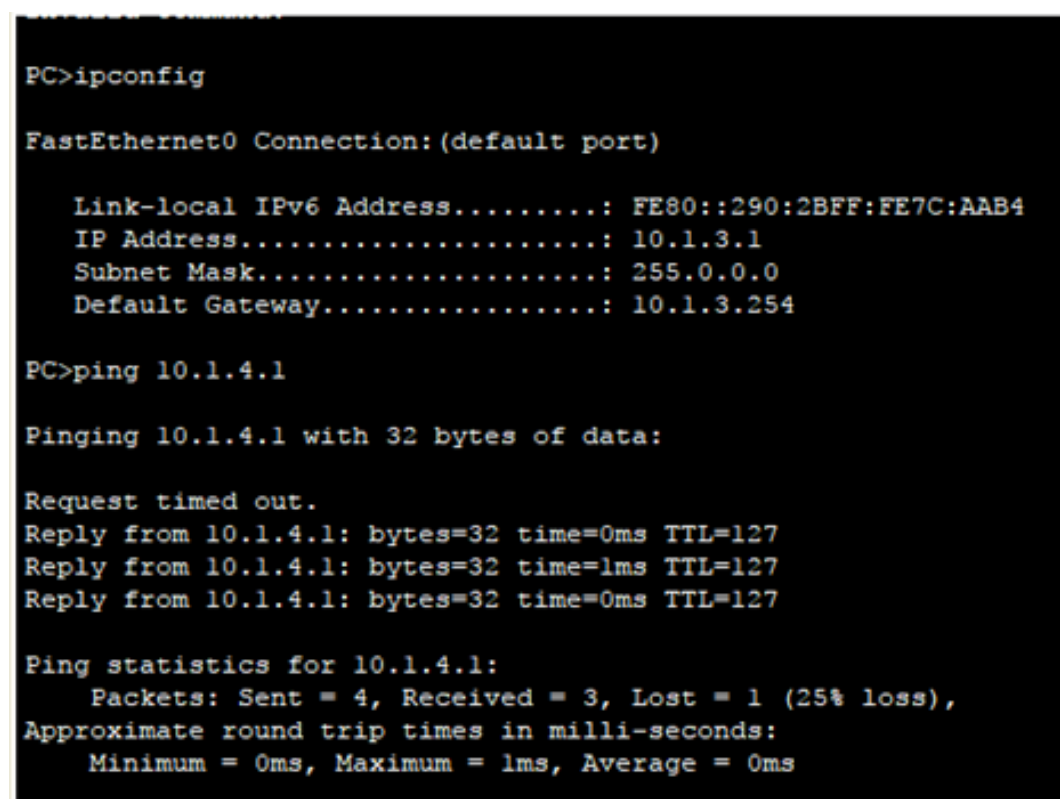

Figure 4 ping test

The figure above shows the PC0 host information. The network between PC0 and PC1 can access each other.

\subsection{OSPF Protocol Configuration Verification}

Work subnets and guest subnets belong to different network segments and need to communicate through routing functions. The router runs OSPF routing protocols. Verify the OSPF protocol configuration information. Verify the OSPF protocol configuration information with the PC1 IP address 10.1.3.1 in the PC subnet 10.1.3.1 in the PING guest network. The result is shown in Figure 5 .

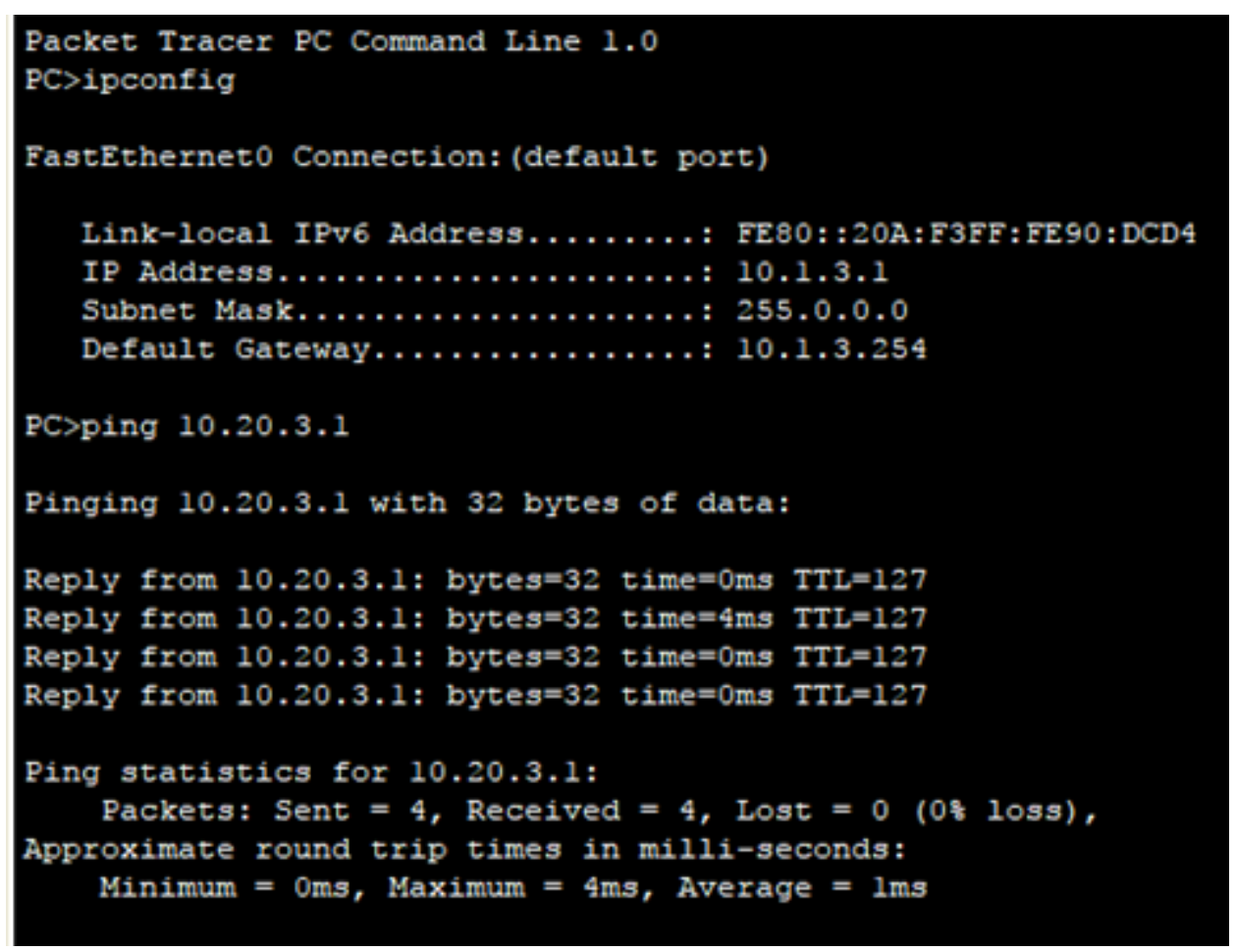

Figure 5 Verifying OSPF

The figure above shows that different VLANs can communicate with each other and OSPF routes are configured correctly. 


\section{Conclusion}

Hotel network planning and design is a complicated process involving many aspects of knowledge. In this design, the hotel network was planned and designed in multiple directions from the design of the hotel's core network to the procurement of equipment and the implementation of hotel network cabling facilities. With the development of the information age, the Internet has become an indispensable infrastructure in life. In order to cater to the future development trend and ensure the hotel industry has a good prospect in the future development, modern hotels must establish excellent network facilities to meet the needs of customers in order to adapt to the development direction of the future hotel network.

\section{Acknowledgements}

This research was supported by Doctor Initial Funding of Hubei University of Science and Technology (No. 2016-19XB003), the Scientific Research Project of Education Department of Hubei Province under Grant B2018179 and B2017181 and B2018175, the National Natural Science Foundation of China (No. 51479155).

\section{References}

[1] Zawar S., Ather S., Imdad U. Effect of transmission opportunity and frame aggregation on VoIP capacity over IEEE 802.11n WLANs. 8th International Conference on Signal Processing and Communication Systems, 2014:1-7.

[2] Pilosof S., Ramachandran R., Raz D., et al. Understanding TCP fairness overwireless LAN, Proceedings of the IEEE INFOCOM, San Francisco, 2003: 863-872.

[3] Gong M.W., Wu Q., Williamson C. Queue management strategies to improve TCP fairness in IEEE 802.11 wireless LANs, Proceedings of the 4th International Symposium on Modeling and Optimization in Mobile, AdHoc, and Wireless Networks, Boston, 2006: 1-8.

[4] Cheng Y.H., Li Z.S., Zhu L. A bandwidth allocation scheme between uplink and downlink flows in IEEE 802.11 WLAN, Journal of South China University (Natural Science Edition), 2009, 5(37): 17-22.

[5] Abeysekera B.S.H., Matsuda T., Takine T. Dynamic contention window control to achieve fairness between uplink and downlink flows in IEEE 802.11 WLANs, Proceedings of the IEEE Wireless Communications and Networking Conference, Hong Kong, 2007, 3517-3525. 